\title{
MBSR: MIMO Based Sink Relocation for Path Selection in IoT Based WSN
}

\author{
Manasa $P^{a}$, Shaila $K^{b}$ and Venugopal $K R^{c}$ \\ ${ }^{a}$ Research Scholar, VTU-RC, Department of Electronics and Communication Engineering, Vivekananda Institute of \\ Technology, Bengaluru \\ ${ }^{b}$ Professor, Department of Electronics and Communication Engineering, Vivekananda Institute of Technology, \\ Bengaluru \\ ${ }^{c}$ Vice Chancellor, Bangalore University, Bengaluru.
}

\begin{abstract}
Wireless Sensor Networks have extended its functionalities by integrating with the Internet of Things and are highly proficient when it is incorporated with other technologies. This demands communication with lesser energy consumption and one such idea of low energy consumption is low energy path selection by a mobile sink node. Path selection requires a good Signal Interference Noise Ratio (SINR) which is calculated for the positioned mobile sink. Thus, a high-quality transmission path is selected by choosing a path to obtain better SINR. MBSR algorithm considers hierarchical clustering schemes and heads are elected to monitor the network. Heads of the clusters communicate to IoT applications using Multiple Input and Multiple Output Dual Antennas. The proposed MBSR scheme utilizes a path optimization technique and finds a suitable path to establish links to end user and realtime environments. The proposed MBSR mechanism employs three phases of execution. Phase 1: Cluster Creation, Phase 2: SINR Analysis for Path Selection. Phase 3: Data transmission to MIMO Devices and in turn forwards to static sinks that connect to end-user using the internet. MBSR improves transmission quality and the proposed scheme is tested for various network parameters to check transmission quality and is compared with existing methodologies.
\end{abstract}

Keywords: Hierarchical Clustering, IoT, MIMO, Signal Interference Noise Ratio, Wireless Sensor Network.

\section{Introduction}

Internet of Things ( IoT) entrusts the connection of devices to establish communication among various applications. Due to the advancement of technology, WSNs can be easily integrated with other technologies and enable users to access various applications. IoT refers to remote sensing, gathering, and communication of information with the view of establishing a connection between real and computer domain. This provides better opportunities for mankind in accessing valuable resources resulting in effective economic utilization. IoT incorporates various devices for communication establishment from real-world to end-user [1] [2]. The IoT supported WSNs consist of sensing, data collection, accumulation, processing, and a communication unit. Data collection and accumulation of information in IoT devices should consume minimum energy [3].

Clustering in IoT refers to the grouping of IoT devices to collect and accumulate the information. It avoids the extra burden on IoT devices for repeated data transfers. In WSN-IoT, LEACH (Low Energy Adaptive Clustering Hierarchy) and other hierarchical protocols enable designing the routing effectively to achieve the quality of service. Different routing schemes are designed in Cluster Head selection $(\mathrm{CH})$ to reduce the overhead in choosing of $\mathrm{CH}$. Power-efficient routing technologies are in the foremost position for IoT applications. Energy balance in deployed devices helps to prolong the battery life of IoT devices. CHs functioning in the network should always utilize minimum hops to reach the information to sink node [5-6]. 
One of the cooperative transmission schemes such as MIMO (Multiple Input and Multiple Output) helps to achieve energy balancing in the entities of the network. In Energy Harvesting MIMO based schemes, each node's productively works to act as a sender as well as a relay node in response to the source node [7-9]. MIMO is highly tolerant of noise and jitter, so they find it applicable in different sectors of IoT. These MIMOs provide better integration between the real environment and the computerized world. Designing the transmission parameters of MIMO based schemes is of great interest since the quality of transmission is controlled by the delineation of the scheme. One such approach in providing the fruitful communication of IoT devices is the path optimization strategies considered among MIMO devices [10-12]. Minimum Interference has to be achieved during communication which facilitates high quality of services in the network. When the sink considered is mobile, effective strategies have to be planned in the accumulation of the information from all IoT devices to the destination. Movable sink node based on MEMO schemes helps IoT applications to make information available at end users with lesser interference [13-14].

a) Motivation: Thiruchelvi [20] et al., proposed route adjustment mechanisms for a mobile sink based on pair based method in IoT. This work proposes five steps of execution which include network division, $\mathrm{CH}$ selection, repositioning the sink nodes, path adjustments, and MAC scheduling. The first phase includes network division by using Binary Tree Algorithm. The second phase includes $\mathrm{CH}$ selection by assigning weights for the node. The third phase involves the sink relocation schemes and includes pair based schemes for choosing an optimized path. Destination oriented schemes are specified for path adjustments. MAC scheduling is done for pair based scheme by $\mathrm{CH}$ and tends to drain out the energy quickly. Pushpalatha [21] et al., proposed optimized sink positions in WSN, and clusters were formed based on distance and retention of energy. Cat Optimization Algorithm (COA) was adopted for $\mathrm{CH}$ selection based on the power level of the nodes and the results converge in idle $\mathrm{CH}$ selection. During the movement of the node, $\mathrm{CH}$ may become overloaded since updating of the node movement increases overhead and faces higher energy losses. Movable receiver nodes can be adjusted to accept the transmitted data at a higher rate.

b) Contribution: IoT integrated devices impose an optimized path for data delivery and ensure absolute placement of the sink device. MIMO schemes ensure multiple transmissions at a time and require a transmission path with higher SINR. The movable MIMO device is positioned in such a way that it assists the network to obtain higher SINR levels. Higher SINR will enable the transmission with less noise and interference. In this article, the MIMO based schemes is proposed via path optimization. MBSR method addresses the interference issues and adopts an optimized path and decreases data loss in the network. Further, MIMO schemes help the sink node to collect multiple inputs with lesser delay. MBSR employs three phases of operation that include partitioning of the network, calculation of efficient SINR path, and data transmission to the end-user using the internet. WSNs Integration with IoT can be achieved by using optimized path determination. Hierarchical Clusters formed in the MBSR method helps to achieve aggregation of information with less consumption of energy. 
2. The rest of the paper is organized as follows; Section 2 describes the related works about WSNs, mobile sink, MIMO techniques, and path optimization schemes. Section 3 explains the illustration of the proposed MBSR work and its steps of execution. Section 4 demonstrates the block diagram of MBSR The block diagram of the MBSR scheme with its functionalities. Section 5 represents the analyses of the MBSR scheme and performances of network parameters. Finally, in the last section conclusions of the MBSR scheme is being presented.Related Work

Khadidja [15] et al., proposed the Hilbert Curve Trajectory Movement for data reception considering mobile sink in WSNs. The method shows that Hilbert curve movement of the sink effectively utilizes the source. This approach involves sensor deployment in two dimensions and cluster formation based on the Hilbert curve order. Integer Linear Programming technique is used to find suitable virtual rendezvous points in each of the clusters created. In this method, Failure of message reception during mobility of the sink can be avoided. The sink is designated to meet virtual points traversing the optimum curves. Trajectories of various curves are compared and the Hilbert curve improves energy gain. Significant energy saving is obtained by considering the Moore Curve Trajectory path in place of the Hilbert curve. Finding an absolute optimization path curve for the sink node becomes prime and accountable functionality in the work.

Tom [16] et al., implemented a protocol based on a position-aware scheme for mobile WSNs [4]. Blind forwarding is used in the decision making of transferring data packets at the receiver node. The priories that are set for transmission are based on the location of nodes from the sink node. Considering the priorities set, the receiver node either forwards the data packet to the next-hop node or discards it. This technique is designed to serve the routing using a collision-free MAC layer. The routing schemes are highly adaptable and robust in its functionality. Few of the network parameters performances are verified by using the global TDMA MAC layer. Reliable and fast transmission can be achieved using this protocol. Low end-to-end delay and a lesser amount of energy consumption are achieved. This technique is implemented by deploying a lesser number of sensor nodes and work can be enhanced by employing ground vehicles.

Range [17] et al., proposed the transmission distance adjustment for multi-sink in the WSNs and the various factors such as the energy consumption, optimization techniques are described. Bigger linear networks are divided into smaller linear networks and sinks are designated as $\mathrm{CH}$. Path selection strategy employs a Multidimensional Multiple-choice Knapsack methodology that effectively selects the node for further data transmission. Heuristic algorithms are considered for resource assignments. Each of the sensor nodes discovers the transmission status of the next-hop node before transmission is initiated to balance the overall energy consumption. This method is compared with other schemes such as direct path and multi-hop path transmission scheme. This technique is suitable when the network is divided based on the multiple sinks.

Yuyang [18] et al., discussed a novel methodology for obtaining higher energy efficiency in WSNs. The enhanced Multiple Inputs and Multiple-Output (MIMO) method defined as Cooperative MIMO (CMIMO) is adapted for data transmission. CMIMO adapts spatial modulation(SM) scheme that enhances energy the efficiency of the system. This multidimensional approach enhances spectral competency. A study of Single bit transmission using CMIMOSM shows that it is energy efficient when compared with conventional methods. Further, work is extended to employ the technique for multi-hop clustered networks. Here, optimizing the hop distance technique is addressed since equidistant hop performance in terms of energy consumption is more.

Thair [19] et al., presented an enhancement for centralized monitoring system using artificial intelligence. Customizing the network by adapting mobile sink in an IOT helps the network to achieve network scalability and prolongs lifetime. The protocol designs are the most favorable specifications of data collection points, network scheduling, and path selection for improving network endurance. The technique inherits Genetic Algorithm and Particle Swarm Optimization techniques when large scale IoT networks are considered. This method accomplishes 
cloud services for optimal selection of clusters, paths, and data gathering points. The entire network is optimally scheduled to balance the energy usage. This method obtains extensive improvement in terms of the number of packets delivered at the sink node but the method doesn't consider the synchronization of sinks when multiple sinks are adapted in the network.

\section{Problem Statement}

In WSN-IoT integrated network during the transmission from sender to end-users poses challenges. The challenges include minimum energy usage for data transmission, good SINR, low latency, and high packet delivery. Interference and noise get added during the data transfer and failure of data packets occurs.

The main objective of the work is to:

(i) Select a path with good SINR

(ii) Improve the network parameters such as Throughput, Packet Delivery Ratio, Energy Consumption, Delay, and Number of Alive Nodes.

\section{Proposed MIMO Based Sink Relocation (MBSR) Technique for Path Selection}

The proposed MBSR algorithm consists of the random deployment of nodes corresponding to nodal points of IoT devices. Communication in the IoT platform requires the grouping of devices and groups formed are called clusters of the network. Clusters created to initiate the process by choosing the leading node in each cluster, based on the vitality status of the Heads. So, each cluster adopts one prime node $(\mathrm{CH})$ to take the responsibility of monitoring the functionality of clusters created. Participants of the cluster, communicate their data to $\mathrm{CH}$ selected via single-hop communication. The mobile sink is assumed to function based on MIMO technique. The sink node is considered to have an exceptional energy and the MIMO approach considers the sink node to receive data from multiple CHs at a time. To receive data from $\mathrm{CHs}$, movable sink acquires an optimized path to make $\mathrm{CHs}$ communicate to sink with minimal hop counts and with less signal interference noise.

MSBR algorithm consists of transmitting and receiving antennas which are MIMO based as shown in Figure 1. These antennas are capable of receiving multiple inputs and connect to multiple output devices. CHs transmit to MIMO antenna based sink node which is placed in a location favorable to achieve higher SINR value. The receiving antennas provide a connection to the static sinks. Due to IoT integration technology, data is received by the end-user via the internet. 


\section{$2^{\text {nd }}$ International Conference on IoT, Social, Mobile, Analytics and cloud Computational Vision and}

Bio-Engineering (ISMAC-CVB 2020)

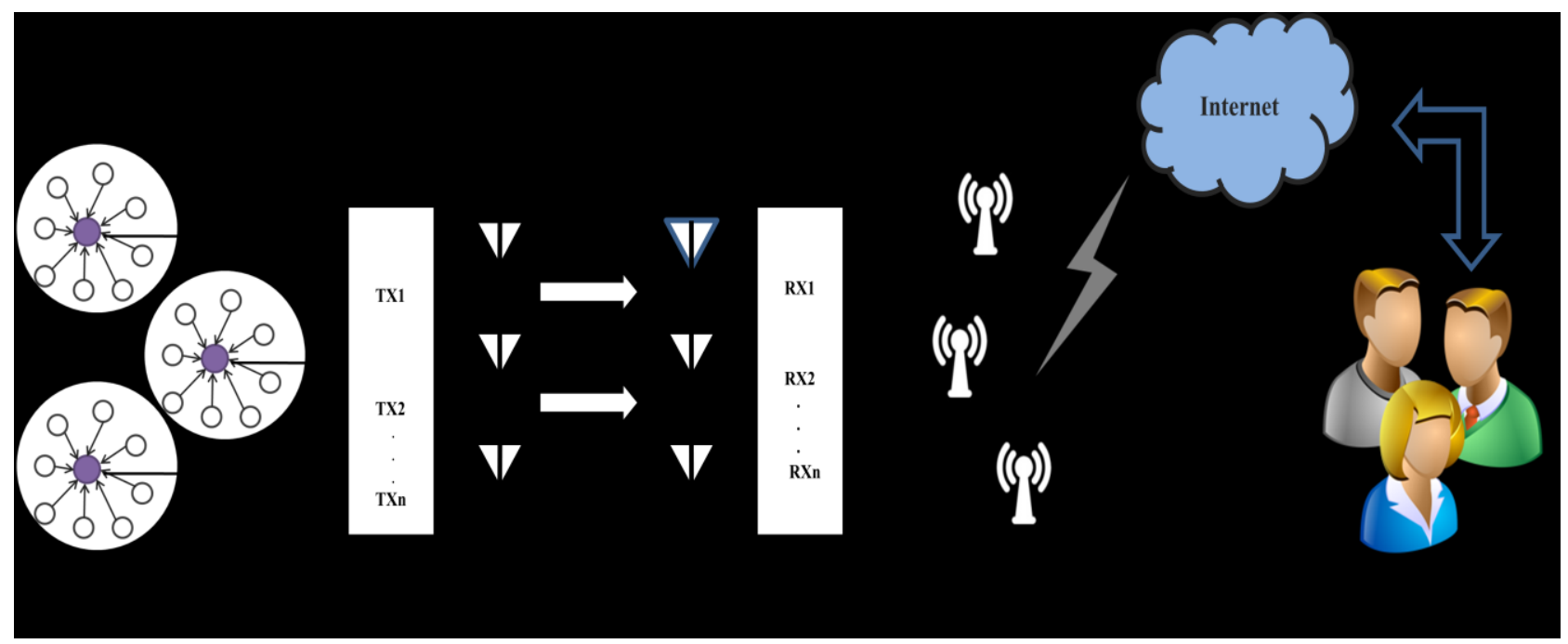

Fig 1: Block Diagram for MBSR Algorithm

Data communication using the MBSR algorithm takes place in three phases of operation Phase 1: Network Partition and cluster creation. Phase 2: Path Optimization and SINR calculation. Phase 3: MIMO Data Transmission as shown in Figure 3.

4.1 Phase 1: Network Partition and Cluster Creation: During this phase of the MBSR algorithm, the area considered for communication is partitioned into different clusters based on the geographical alignment of nodes in the network. The partitioned areas of the network form clusters of a network. Hierarchical methods are beneficial in accumulating and transferring of information since they can overcome redundant data transfers in the network. Energy-saving can be achieved by electing a monitoring entity that has more energy when compared with other members of clusters and performs the task of data fusion. The hierarchical strategy used in partitioned head selection $(\mathrm{CH})$ is LEACH which considers the highest retained energy as a parameter to select the area head. One $\mathrm{CH}$ is elected in each of the partitioned areas that have the highest energy when compared with other nodes in a given cluster. To avoid malicious node as the area head validation of elected $\mathrm{CH}$ must be done. This task is achieved by performing an authentication check of CHs based on their ID and energy level. This is one of the prime factors in avoiding transmission to malicious nodes. Figure 2 shows the Hierarchical Clustering adopted in the MBSR scheme.

4.2 Phase 2: Path Optimization and SINR Calculation: In the network, mobile sink nodes are enabled with the MIMO scheme to collect data from all CHs. MIMO device consists of an omnidirectional antenna that is capable of receiving the transmitted data from surrounding devices in any direction. Path optimization is the efficient path created to MIMO devices to receive inputs from CHs. MIMO enabled mobile sink node that are capable of locomotion in the network requires a proper location to receive data with lesser energy consumption. The shortest distance algorithm is utilized to calculate the distance for each $\mathrm{CH}$ and MIMIO node. Thus, overall energy consumption is calculated based on the distance. It is highly recommended to carry an SINR check of the path selected since noise interference may occur during transmission. This ratio reveals transmission quality by knowing the channel capacity of transmission. SINR is obtained for a selected path and is compared with the threshold SINR. If the obtained SINR is not acceptable, alternate path calculations and a new set of SINR computations are performed to obtain the most optimized path for data transfers. 


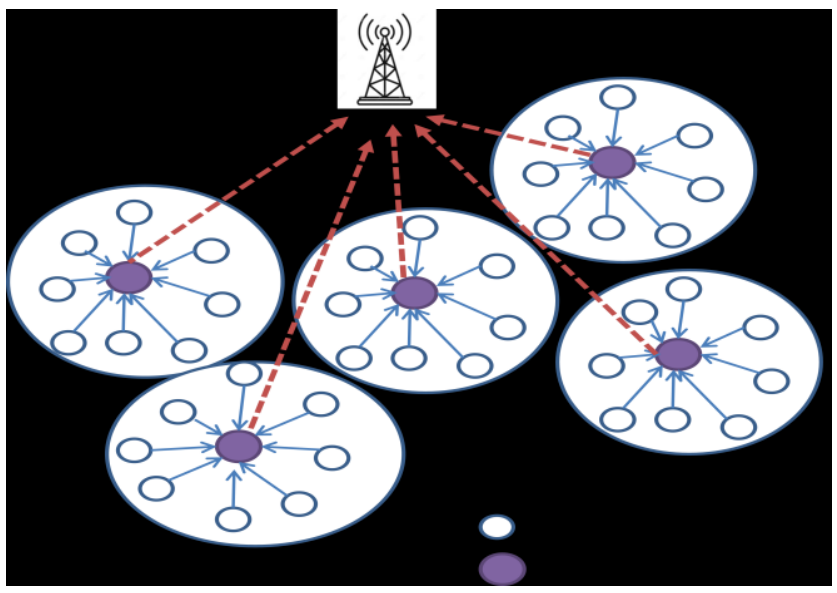

Fig 2: Hierarchical Clustering

4.3 Phase 3: MIMO Data Transmission Phase: After the selection of an optimized path connecting different $\mathrm{CHs}$, the MIMO node receives multiple transmissions from different CHs. MIMO connects to various multiple output devices such as static sinks of numerous applications. The variety of applications in the IoT environment connects to the MIMO device and obtains information of all the deployed nodes in the network. Thus, successful MIMO data transmission is achieved.

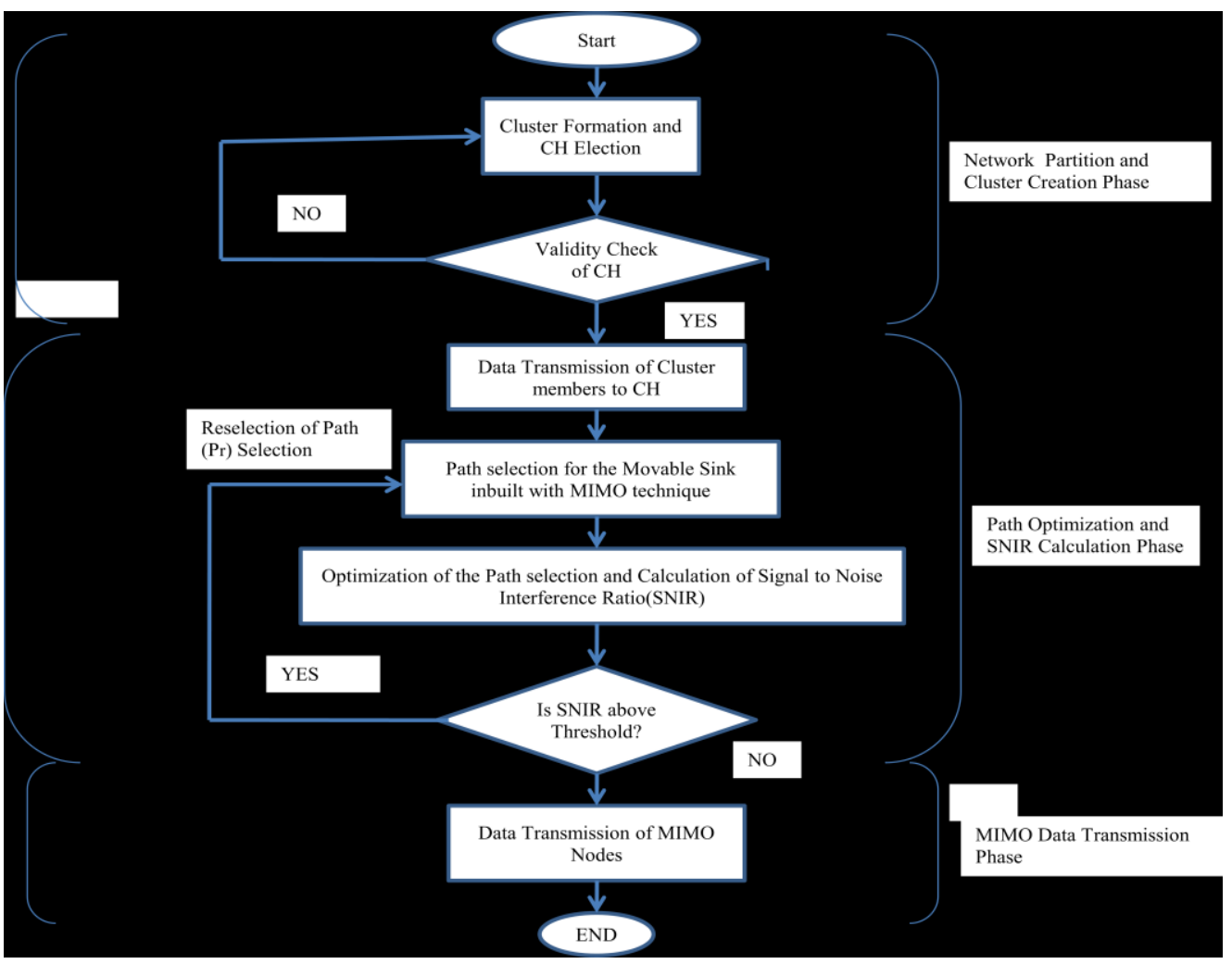

Fig. 3 MBSR Flow chart 
4.4 SINR Analysis in MBSR Scheme: SINR corresponds to the received signal quality of the network. This varies depending on the propagation path and position of sink transmitter and receiver antennas. Random variations may occur that causes SINR to vary. SINR is given by,

$$
\text { SINR }=\frac{\text { Signal Power }}{\text { Interference+Noise }}
$$

Interference may occur by the neighboring cells and SINR for the homogeneous network is given by,

$$
\operatorname{SIR}_{\mathrm{x}}=\frac{\mathrm{P}_{\mathrm{r}, \mathrm{x}}}{\Sigma_{Y} \mathrm{I}_{\mathrm{y}}+\mathrm{C}_{\mathrm{o}}}
$$

Where, $\mathrm{P}_{\mathrm{r}, \mathrm{x}}=$ signal power of the desired node, $\mathrm{I}_{\mathrm{y}}=$ total signal interference of all neighboring nodes that includes cotier interference. $y=$ number of nodes that are employed. $C_{o}=$ thermal noise power. In equation 2 , the second term shows interference due to Lp. The SINR for the heterogeneous network is given by,

$$
\operatorname{SIR}_{\mathrm{x}}=\frac{\mathrm{P}_{\mathrm{r}, \mathrm{x}}}{\sum_{\mathrm{y}=\mathrm{x}}^{\mathrm{y}} \mathrm{I}_{\mathrm{y}}+\mu \sum_{\mathrm{y} \neq \mathrm{x}}^{\mathrm{Y}} \mathrm{LLp} \sum_{\mathrm{s}=1}^{\mathrm{S}} \mathrm{P}_{\mathrm{r}, \mathrm{x}, \mathrm{y}}+\mathrm{C}_{\mathrm{o}}}
$$

Where, $P_{r, x, y}=P_{t, x} h_{x, y} G_{x} d_{x, y}^{-c}$, $h x=$ fading channel gain between user desired node and the user. Lp = number of various low power nodes and $G_{x}=$ low power node antenna gain value. $C=$ path loss factor in exponent $\mu=1$ for heterogeneous and $\mu=0$ for the homogeneous network. Thus, the signal to noise interference is calculated and an optimized path is selected for transmission.

\section{Performance Evaluation}

Performance Evaluation of MBSR and MCMIMO technique is done using the NS2 tool. MBSR schemes are compared with the existing MCMIMO technique which is a multichannel based network. Cluster heads are selected by assigning weights to all the deployed nodes. The link connecting different nodes is verified with the energy level to enable interrupted services but, the link quality concerning interference and noise is not considered. Our proposed method MBSR scheme utilizes channel quality measurement metrics to determine seamless transmission to the sender. 


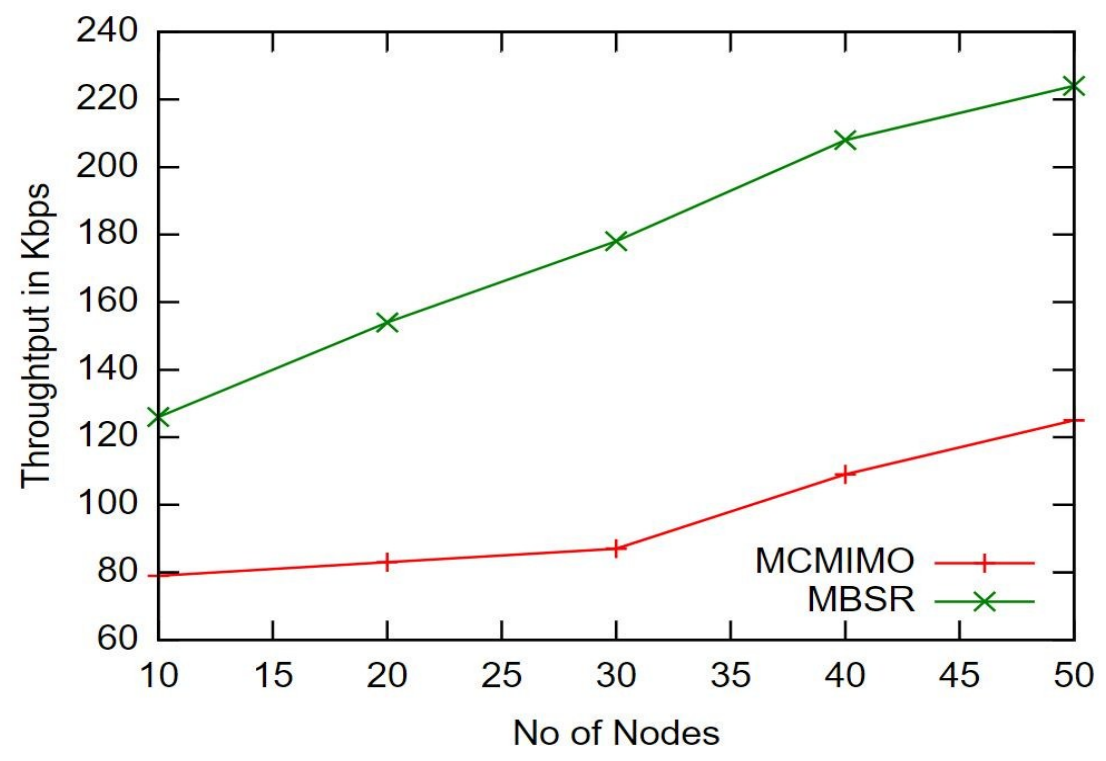

Fig. 4 Throughput vs. Number of Nodes

SINR is the direct measure of transmission quality of communication. It enables successful communication between the sender and the receiver. MBSR incorporates the mechanism to have a high-quality channel formed between transmitting and receiving ends. MCMIMO reduces only the amount of data delivery whereas MBSR addresses the techniques for fading channel. As the number of nodes increases, more nodes are available for routing, and throughput increases as shown in Figure 4. Compared to the MCMIMO scheme, MBSR presents a high throughput value since the number of packets delivery is increased by $13 \%$.

Figure 5 shows the analysis of the delay parameter for the MCMIMO scheme and the MBSR scheme. Since highquality channels support for early transmission, the delay is comparatively less compared to MCMIMO schemes. End-to-end delay is plotted for MCMIMO and MBSR techniques. Figure 6 represents the comparison of MCMIMO with MBSR techniques concerning the packet delivery ratio. This parameter represents successful packet transmission using MIMO schemes. However, during channel fading, some of the packets may be lost and result in a reduced Packet Delivery Ratio. The possibility of channel fading in MCMIMO is high because the noise interference avoidance mechanisms are not adopted. 


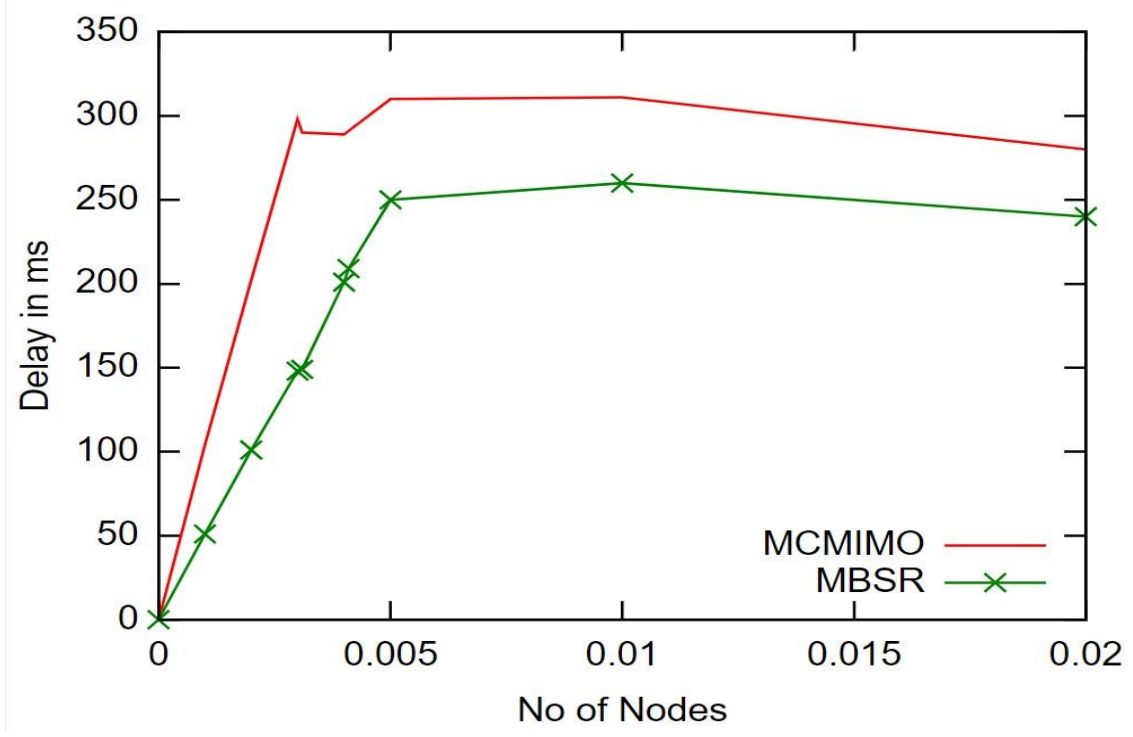

Fig. 5: Delay in ms for varying Numbers of Nodes

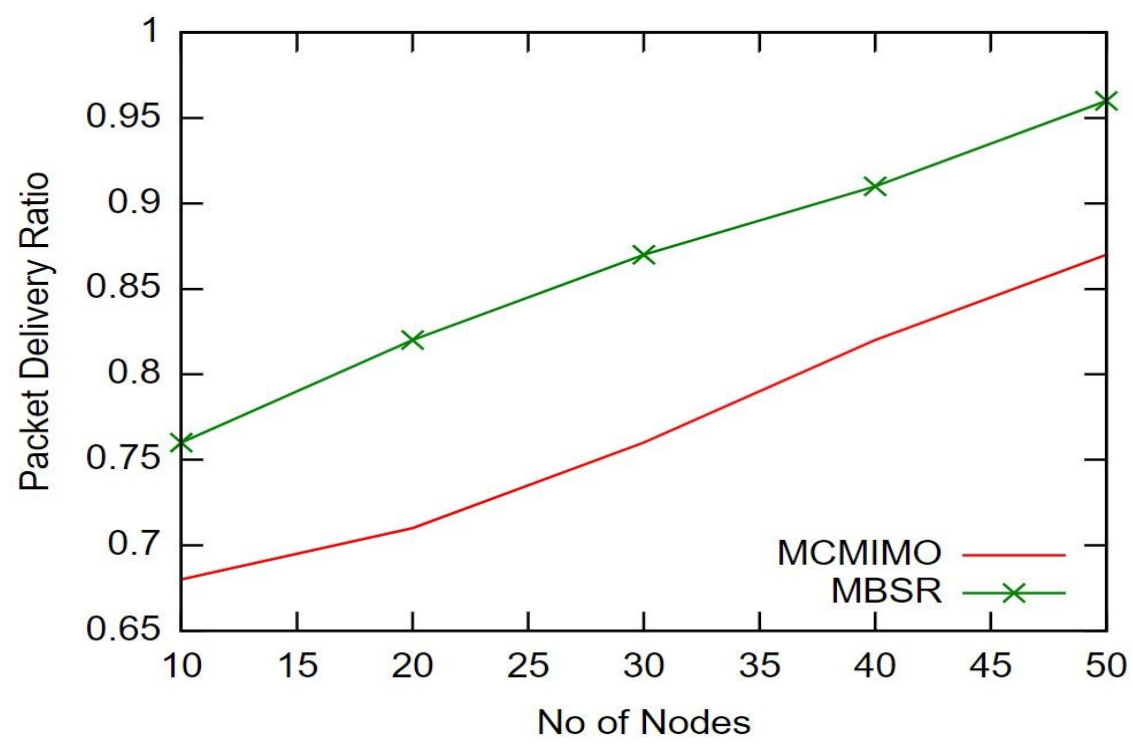

Fig. 6: Packet Delivery Ratio for varying Number of Nodes

Figure 7 represents the count of packets lost during transmission in the MBSR technique and is compared with the MCMIMO scheme. During the occurrence of interference in the channel, degradation of bandwidth occurs resulting in losing some of the nodes when such channels are selected for transmission. The graph shows an increase in packet loss for an increasing number of nodes. As the number of nodes increases, contention in the channel increases resulting in reduced data-carrying packets in the system. The determination of movable sink location plays a major role in overcoming packet delivery failure in the MBSR method. The mobile sink is in the appropriate position for which SINR is of greater value and this position of sink is taken as a reference to find an optimized path. 


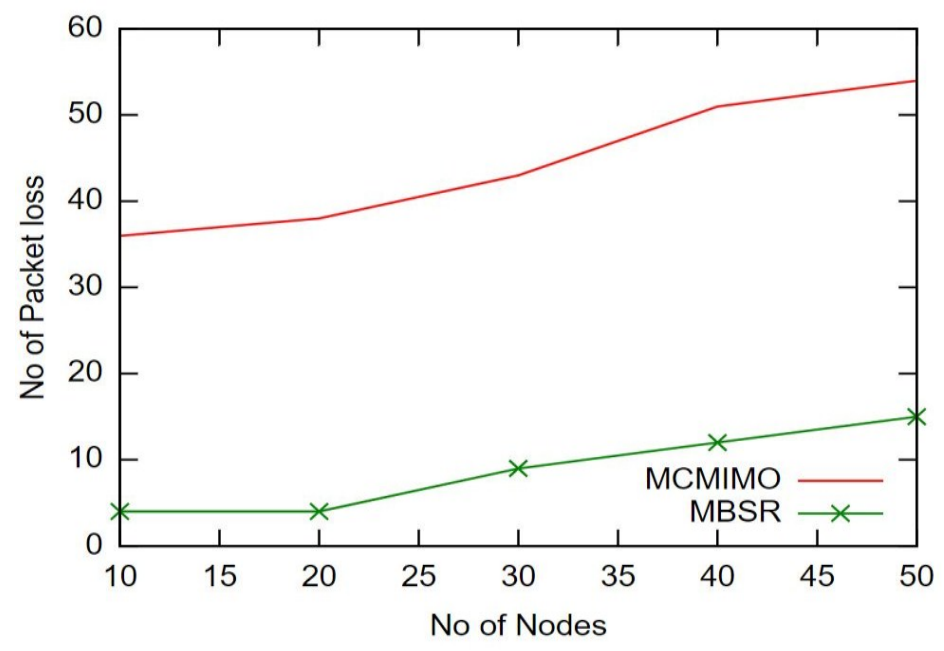

Fig. 7: Number of Packet loss for varying Numbers of Nodes

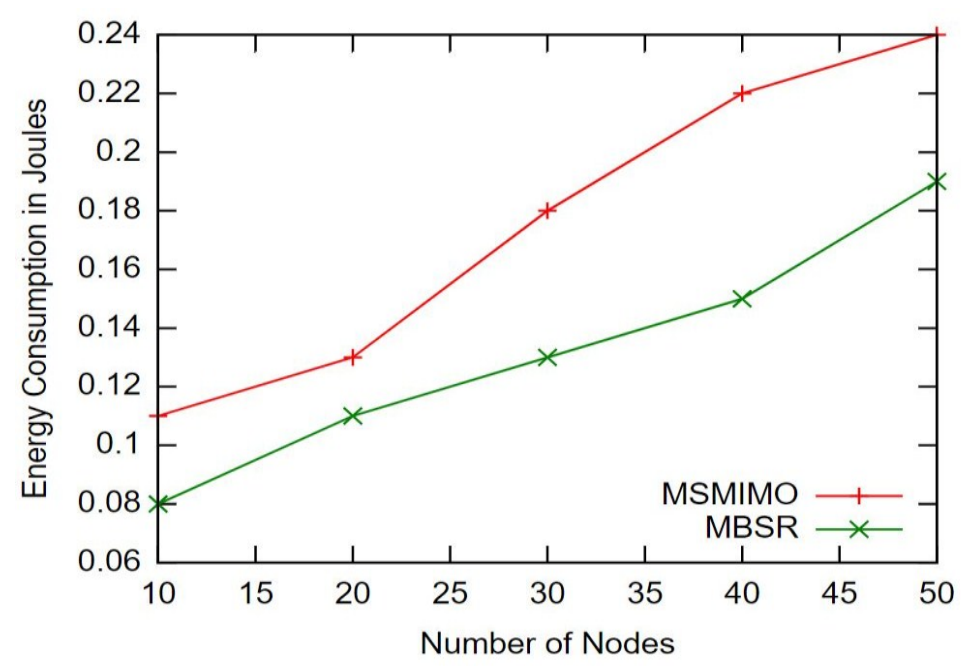

Fig. 8: Energy Consumption for varying Number of Nodes

Figure 8 shows the comparison of MSMIMO and MBSR schemes concerning energy consumption for a varying number of nodes. Energy consumption in communication is directly proportional to noise and interference effects in the network. Since MSMIMO retransmits the packet during fading effects in the channel, more energy is being consumed when compared with MBSR techniques. Energy consumption in the MBSR technique is reduced by $18 \%$ compared with the MSMIMO technique.

\section{Conclusion}

The proposed scheme MBSR can be integrated into IoT by adopting MIMO functionalities and helps to achieve network efficiency. The movable sink node reaches various locations of the network to receive a high amount of data with less noise interference. Path optimization techniques utilize standards of SINR to overcome data degradation in the network. Clusters that are formed during network partition in MBSR reduce redundant data transmission. Network parameters such as count of the packet loss, Delay, PDR, Energy Consumption, and 


\section{$2^{\text {nd }}$ International Conference on IoT, Social, Mobile, Analytics and cloud Computational Vision and Bio-Engineering (ISMAC-CVB 2020)}

throughput of the proposed MBSR are correlated with the techniques of MCMIMO. The packet loss in the network is reduced by $30 \%$ by introducing a movable sink and by utilizing techniques of low SINR in the network. Path optimization techniques designed to obtain low interference helps in achieving the improvement over MCMIMO. MBSR technique can be further improved in terms of the scalability of the network.

\section{REFERENCES}

[1] JinWang, Jiayi Cao R, Simon Sherratt Jong Hyuk Park, Jiayi Cao,“An Improved Ant Colony Optimization-Based Approach with Mobile Sink for Wireless Sensor Networks” The Journal of Supercomputing, vol. 74, no. 12, pp. 6633-6645, 2018.

[2] Nayak, P and Reddy, C.P., "Bio-inspired Routing Protocol for Wireless Sensor Network to Minimise the Energy Consumption", IET Wireless Sensor Systems, vol. 10, no. 5, pp.229-235, 2020.

[3] Zhang, Y. and Wang, Y., "A Novel Energy-Aware Bio-inspired Clustering Scheme for IoT Communication”, Journal of Ambient Intelligence and Humanized Computing, pp.1-10, 2020.

[4] Jagriti D.K, Lobiyal, "Energy Consumption Reduction in SMAC Protocol for Wireless Sensor Network", Procedia Computer Science, vol. 143, pp. 757-764, 2018.

[5] Ruilong Deng, Shibo He and Jiming Chen, "An Online Algorithm for Data Collection by Multiple Sinks in Wireless Sensor Networks", IEEE Transactions on Control of Network Systems, vol. 5, no. 1, pp. 93-104, 2018.

[6] J. Chang, L. Zhang, and B. Zeng, "Data Gathering Algorithm for Mobile Sink Based on the Global Delivery Latency Minimization", Chinese Journal of Sensors and Actuators, vol. 29, no. 2, pp. 264-270, 2016.

[7] C. Wan, J. Chang, and L. Zhang, "Dynamic Path Planning Design of Mobile Sink for Single Region Bursty Traffic", Journal of Computer Science, vol. 44, no. 5, pp. 61-65, 2017.

[8] Saranya, S. Shankar, G. R. Kanagachidambaresan, "Energy Efficient Clustering Scheme (EECS) for Wireless Sensor Network with Mobile Sink", Journal of Wireless Personal Communications, vol. 100, no. 4, pp. 1553-1567, 2018.

[9] Behera, T.M, Mohapatra, S.K, Samal, U.C, "Residual Energy Based Cluster-head Selection in WSNs for IoT Applications", Journal of IEEE Internet of Things, vol. 6, no. 3, pp. 5132-5139, 2019.

[10] Wang, Jin, Jiayi Cao, Sai Ji, and Jong Hyuk Park. "Energy-Efficient Cluster-Based Dynamic Routes Adjustment Approach for Wireless Sensor Networks with Mobile Sinks", The Journal of Supercomputing, vol. 73, no. 7, pp. 3277-3290, 2017.

[11] Chaibrassou and A. Mouhsen and I. Lagrat, "Efficient and Distributed Clustering Scheme with Mobile Sink for Heterogeneous Multi Level Wireless Sensor Networks", Journal of Theoretical and Applied Information Technology, vol. 63. no. 3, pp. 597-604, May 2014.

[12] Zhang, Deyu, Zhigang Chen, Haibo Zhou, Long Chen, and Xuemin Sherman Shen, "Energy-Balanced Cooperative Transmission Based on Relay Selection and Power Control in Energy Harvesting Wireless Sensor Network", Journal of Computer Networks, 104, pp. 189-197, 2016.

[13] Yuste-Delgado, Antonio-Jesus, Juan-Carlos Cuevas-Martinez and Alicia Trivino-Cabrera. "A Distributed Clustering Algorithm Guided by the Base Station to Extend the Lifetime of Wireless Sensor Networks", Journal of Sensors, vol. 20, no. 8, pp 2312, 2020.

[14] Yuan, Yong, Zhihai He and Min Chen. "Virtual MIMO-Based Cross-Layer Design for Wireless Sensor Networks", Journal of IEEE Transactions on Vehicular Technology, vol. 55, no. 3, pp 856-864, 2006.

[15] Fellah, Khadidja, and Bouabdellah Kechar. "New Approach Based on Hilbert Curve for Energy Efficient Data Collection in WSN with Mobile Sink", Journal of IET Wireless Sensor Systems, vol. 10, no. 5, pp. 214-220, 2020.

[16] Hayes T, Ali FH, "Location Aware Sensor Routing Protocol For Mobile Wireless Sensor Networks", Journal of IET Wireless Sensor Systems, vol. 6, no.2, pp. 49-57, 2016.

[17] Bing, Su and Zhang Yujing. "Energy Efficiency In Multi-Sink Linear Sensor Network With Adjustable Transmission Range." In 2016 8th IEEE International Conference on Communication Software and Networks, pp. 462-466, 2016.

[18] Peng, Yuyang, and Jaeho Choi. "A New Cooperative MIMO Scheme Based on SM for Energy-Efficiency Improvement in Wireless Sensor Network", Journal of The Scientific World, Volume 2014, Article ID 975054, 10 pages, 2014.

[19] Al-Janabi, T. A., \& Al-Raweshidy, H. S, “A Centralized Routing Protocol with a Scheduled Mobile Sink-Based AI for Large Scale IIot”, IEEE Sensors Journal, vol. 18, no. 24, pp. 10248-10261, 2018.

[20] Thiruchelvi, A, and Natesapillai Karthikeyan. "Pair-Based Sink Relocation and Route Adjustment in Mobile Sink WSN Integrated IoT", Journal of IET Communications, vol.14, no.3, pp. 365-375, 2019.

[21] Pushpalatha, G. Kousalya, "A Prolonged Network Life Time and Reliable Data Transmission Aware Optimal Sink Relocation Mechanism”, Journal of Cluster Computing, vol. 22, no. 3, pp. 1-10, 2019. 